Editorial

JMCJMS

\title{
Challenges of Medical Education in Nepal
}

\author{
Shankar PR \\ Editor, JMCJMS
}

Medical Education in Nepal is a recent phenomenon with the Institute of Medicine, the first medical school in the country admitting the first batch of undergraduate medical students in 1978 AD. In the decades from 1990 to 2010 AD a number of new medical schools have opened in the country. Many medical schools also run postgraduate programs in different subjects. Recently certain schools have also started postdoctoral (DM and MCh) courses. At present the medical schools in the country are in a position to meet a large percentage of its medical manpower needs. The majority of medical schools in Nepal are in the private sector and in addition to Nepalese students also admit students from other countries, both from South Asia and from outside the region. Despite all round progress there are also many challenges faced by medical schools and medical education in Nepal. In this editorial the author will briefly examine some of these.

Ensuring adequate infrastructure standards: The regulatory bodies, the Universities and the Nepal Medical Council (NMC) have fixed infrastructure requirements for colleges depending on the student intake. However, infrastructure standards vary widely between schools. Also in certain departments like in Pharmacology the equipment and facilities prescribed are directed towards animal experimentation while the emphasis in Nepal is on small group work and teaching students to use essential work and teaching students to use essential medicines rationally. Physical separation of the basic science and clinical science campuses creates lots of problems. The interaction between basic and clinical sciences is less and faculties of basic sciences find it difficult to be involved in clinical activities.

Meeting faculty requirements: In the initial stages (1990s and early 2000) faculty were recruited from neighboring countries mainly from India. Recently a number of new medical schools have started in India and the remuneration for medical school faculty has increased substantially. Recently at the junior levels trained faculty members are being created in Nepal. Also recently NMC and the Universities have allowed dental (BDS) graduates to apply for $\mathrm{MD}$ in Basic Sciences after completing a bridge course to meet the demand of Basic science faculty requirements.

Quality of student intake: Medicine is an academically demanding discipline. Hence student selection for the course is important. Nepalese scholarship students are selected through an entrance examination conducted by the Ministry of Education while self-financing students have to qualify (obtain 50\% of marks) in exams conducted by Universities. I am of the opinion that the University should also rank the students according to marks obtained in the entrance exam and then students can be counseled regarding which college to apply. Objective information about education, infrastructure and other standards at different institutions in the country is not available. Foreign students only have to obtain $50 \%$ of marks in science subjects and English in the $12^{\text {th }}$ standard exam. Obtaining marks in school leaving exams is easy and most students manage to easily obtain the required percentage. Another problem I have noted is many students do not have the required proficiency in written and spoken English and struggle especially in 
the initial years. A test of proficiency in the English language, the medium of instruction could be considered. Also selection of students is primarily determined by financial consideration and often academic leaders do not have much input into student selection.

Ensuring quality of teaching and assessments:

The quality of teaching and of faculty varies between medical schools. Certain schools have a well-designed academic program followed throughout the year. In other schools teachinglearning activities are not of adequate standards. As an example in my subject of clinical pharmacology at KIST Medical College we have a structured program with over 25 sessions being conducted over two years. Part of this neglect may stem from our emphasis on theory and disregard for practice. Senior professors usually concentrate only on theory classes leaving the practical sessions to junior faculty and postgraduates. Ensuring fairness of assessments and curbing malpractice is also a major challenge for universities and they often are not completely successful in this task.

Matching manpower with the needs of the country's health system:

Nepal like many developing countries suffers from a serious shortage of medical manpower especially in rural areas. Most self-financing students in private schools pay a large amount as tuition and other fees. The amount can vary between Nepalese Rupees of 25 to 45 lakhs depending on the school and other criteria. After paying such a heavy amount issues of return of investment crops up and graduates are likely to migrate to developed nations. Among the many students I have taught over the years a large percentage have migrated.
Scholarship students in private schools have to serve in rural Nepal for a period of two years after graduation. A common complaint is that their course of study does not prepare them adequately for the challenges of working in rural areas.

In this brief editorial I have examined few of the challenges facing medical education in the country. There are also other challenges which have to be addressed. Addressing these challenges can ensure high quality of medical education in the country and ensure that the schools attract students from abroad and at the same time meet the country's pressing healthcare needs.

NB: The Publisher and editorial board is not responsible about the information provided in the articles. Authors are responsible for the same.

Published by:
Research Department, Janaki Medical
College
Ramdaiya, Janakpurdham
Nepal

